\title{
Instrumentos utilizados na avaliação psicológica no tratamento da obesidade: revisão integrativa
}

\author{
Instruments used in psychological assessment in the treatment of Obesity: an integrative review \\ Instrumentos utilizados en la evaluación psicológica en el tratamiento de la obesidad: una revisión
}

integradora

Recebido: 21/12/2021 | Revisado: 29/12/2021 | Aceito: 03/01/2022 | Publicado: 05/01/2022

\author{
Vanderson Garcia da Silva \\ ORCID: https://orcid.org/0000-0003-1101-5163 \\ Universidade Federal Fluminense, Brasil \\ E-mail: vandersongarcia@id.uff.br \\ Fátima Helena do Espírito Santo \\ ORCID: https://orcid.org/0000-0003-4611-5586 \\ Universidade Federal Fluminense, Brasil \\ E-mail: fatahelens@mail.com \\ Maria de Nazaré de Souza Ribeiro \\ ORCID: https://orcid.org/0000-0002-7641-1004 \\ Universidade do Estado do Amazonas, Brasil \\ E-mail: mnribeiro2@gmail.com \\ Juliana Lopes Fernandes Massapust Pestana \\ ORCID: https://orcid.org/0000-0002-7228-0596 \\ Universidade do Estado do Amazonas, Brasil \\ Universidade federal do Rio de Janeiro, Brasil \\ E-mail: julianalmassapust@gmail.com
}

\begin{abstract}
Resumo
Os instrumentos utilizados na avaliação psicológica no tratamento clínico ou cirúrgico da obesidade tem grande relevância no resultado e processo da avaliação. Identificar personalidade, ansiedade, depressão e compulsão alimentar é fundamental, pois são fatores que podem afetar diretamente na manutenção do peso e adesão ao tratamento. O objetivo desta pesquisa foi analisar a literatura científica sobre instrumentos utilizados na avaliação psicológica da pessoa obesa. Trata-se de uma revisão integrativa realizada nas bases de dados: BVS, Medical Subject Headings (MeSH), da National Library, com os seguintes descritores controlados: "psychometrics", "psychological test", "Psychological Assessment", "psychologist", "obesity", "overweight" e o descritor não controlado "psychological assessment" e os operadores booleanos "OR" e "AND". Ao final das buscas, 14 publicações atenderam aos critérios de análise e foram selecionadas para o estudo. Diante dos estudos selecionados, encontramos 52 ferramentas utilizadas na avaliação, dentre eles, os mais utilizados nos programas de perda de peso foram respectivamente o Beck Depression Inventory - BDI (Inventário de Depressão de Beck); o Binge Eating Scale - BES (Escala de Compulsão Alimentar Periódica - TCAP); e a Escala Hospitalar de Ansiedade e Depressão - HADS. Essa pesquisa nos dá evidências importantes para elaboração instrumentos específicos do tratamento da obesidade no Brasil.
\end{abstract}

Palavras-chave: Psicometria; Avaliação psicológica; Psicólogo; Obesidade; Excesso de peso.

\begin{abstract}
The instruments used in psychological assessment in the clinical or surgical treatment of obesity have great relevance in the assessment result and process. Identifying personality, anxiety, depression and binge eating is essential, as they are factors that can directly affect weight maintenance and treatment adherence. The aim of this research was to analyze the scientific literature on instruments used in the psychological assessment of obese people. This is an integrative review carried out in the following databases: BVS, Medical Subject Headings (MeSH), from the National Library, with the following controlled descriptors: "psychometrics", "psychological test", "Psychological Assessment", "psychologist", "obesity", "overweight" and the uncontrolled descriptor "psychological assessment" and the Boolean operators "OR" and "AND". At the end of the searches, 14 publications met the analysis criteria and were selected for the study. From the selected studies, we found 52 tools used in the evaluation, among them, the most used in weight loss programs were, respectively, the Beck Depression Inventory - BDI (Beck Depression Inventory); the Binge Eating Scale - BES (Periodic Binge Eating Scale - TCAP); and the Hospital Anxiety and Depression Scale - HADS. This research provides us with important evidence for the development of specific instruments for the treatment of obesity in Brazil.
\end{abstract}

Keywords: Psychometry; Psychological assessment; Psychologist; Obesity; Overweight. 


\begin{abstract}
Resumen
Los instrumentos utilizados en la evaluación psicológica en el tratamiento clínico o quirúrgico de la obesidad tienen gran relevancia en el resultado y proceso de la evaluación. Identificar la personalidad, la ansiedad, la depresión y los atracones es fundamental, ya que son factores que pueden afectar directamente el mantenimiento del peso y la adherencia al tratamiento. El objetivo de esta investigación fue analizar la literatura científica sobre los instrumentos utilizados en la evaluación psicológica de las personas obesas. Se trata de una revisión integradora realizada en las siguientes bases de datos: BVS, Medical Subject Headings (MeSH), de la Biblioteca Nacional, con los siguientes descriptores controlados: "psicometría", "prueba psicológica", "Evaluación psicológica", "psicólogo", "obesidad", "sobrepeso" y el descriptor no controlado "evaluación psicológica" y los operadores booleanos "OR" y "Y". Al final de las búsquedas, 14 publicaciones cumplieron los criterios de análisis y fueron seleccionadas para el estudio. De los estudios seleccionados, encontramos 52 herramientas utilizadas en la evaluación, entre ellas, las más utilizadas en los programas de adelgazamiento fueron, respectivamente, el Inventario de Depresión de Beck - BDI (Inventario de Depresión de Beck); la Escala de atracones - BES (Escala de atracones periódicos - TCAP); y la Escala Hospitalaria de Ansiedad y Depresión - HADS. Esta investigación nos proporciona evidencia importante para el desarrollo de instrumentos específicos para el tratamiento de la obesidad en Brasil.
\end{abstract}

Palabras clave: Psychometrics; Evaluación psicológica; Psicólogo; Obesidad; Sobrepeso.

\title{
1. Introdução
}

A obesidade é caracterizada pelo acúmulo excessivo de gordura corporal no indivíduo. Suas causas podem estar relacionadas à influência genética, história de aprendizagem, disfunções endócrinas e sedentarismo. Como consequências desta, diversas doenças acompanham à obesidade, como: hipertensão arterial, doenças cardiovasculares e diabetes. (Oliveira et al. 2018).

A Associação Brasileira de Estudo da Obesidade e da Síndrome Metabólica, em suas diretrizes, também, apontou que a obesidade está associada à diminuição da atividade física, aumento da ingestão calórica, o desequilíbrio energético através do consumo de alimentos super calóricos, alta palatabilidade, baixo poder de saciedade, fácil absorção e digestão, e o estilo de vida. (ABESO, 2016)

A Organização Mundial da Saúde (OMS, 2018) também apontou uma crescente da obesidade atribuída às causas multifatoriais. Em 2016, mais de 1,9 bilhão de adultos estavam acima do peso, gerando um problema de saúde pública, ou seja, os dados mostram que 39\% dos adultos com 18 anos ou mais estavam acima do peso em 2016.

Os números são crescentes conforme alguns dados importantes sobre a epidemiologia do sobrepeso e obesidade no Brasil, e que precisam ser enfatizados. Em 27 cidades do país, a frequência do excesso de peso foi de 50,8\%, sendo maior entre homens $(54,7 \%)$ em comparação as mulheres $(47,4 \%)$. Algumas variações ocorreram em algumas cidades, como em São Luís $(41,7 \%)$ para homens e em Cuiabá $(54,9 \%)$ para mulheres. A maior frequência de excesso de peso em homens foi encontrada em Porto Alegre (62,1\%), Macapá (60,8\%) e João Pessoa (59,3\%) e, para as mulheres, em Manaus (52,0\%), Cuiabá (51,0\%) e Campo Grande (50,9\%). Já os índices da obesidade observadas nessas cidades foram respectivamente, no caso dos homens, em Macapá (22,8\%), Cuiabá (21,9\%) e Rio de Janeiro (21,1\%) e, no caso de mulheres, em Cuiabá (22,8\%), Campo Grande (20,5\%) e Rio de Janeiro (20,3\%). As menores frequências de obesidade ocorreram, entre homens, em São Luís (12,3\%), Salvador $(13,1 \%)$ e Belo Horizonte (13,7\%) e, entre mulheres, em Palmas (13,1\%), São Luís (13,9\%) e Macapá (14,2\%). (Mancini et al., 2015)

Com o fim de caracterizar a obesidade, o Índice de Massa Corporal (IMC) é o critério mais conhecido e enfatizado para identificá-la, conforme a Agência Nacional de Saúde Suplementar (ANS). Os valores de IMC iguais ou superiores a $25 \mathrm{~kg} / \mathrm{m} 2$ caracterizam excesso de peso. Entretanto, essa avaliação também pode ser medida por métodos antropométricos, impedância bioelétrica, tomografia computadorizada, ultrassonografia, ressonância magnética, cada um com suas vantagens e limitações.

(Mancini et al. 2015) 
Em suma, o cálculo consiste em dividir o peso (em $\mathrm{kg}$ ) pela altura (em metros) ao quadrado. Um IMC entre 18,5 e 24,9 é considerado saudável para homens e mulheres adultos. Porém, o IMC igual ou acima de 30 já pode ser considerado fator de riscos para as pessoas (ANS, 2017).

O avanço da obesidade desafia a saúde pública no Brasil. A Política Nacional de Promoção da Saúde (PNaPS) tem enfatizado a importância de trata-la, justificada pela crescente nas últimas quatro décadas, mas somente nos últimos 15 anos teve sua prioridade nas políticas públicas, por se associar às Doenças Crônicas Não Transmissíveis (DCNTs). (Dias et al. 2017).

Com o objetivo de conter esse problema, a Organização Mundial da Saúde (OMS), através da estratégia global, tem enfatizado a promoção da alimentação saudável. Para isso, o governo é orientado a desenvolver periodicamente ações, a fim de promover hábitos alimentares saudáveis (PNAN, 2015).

Ainda sobre essa questão, os resultados citados nessa pesquisa corroboram com as estratégias sustentadas durante alguns anos pelos nutricionistas da linha "Nutrição Comportamental" que enfatizam que dietas não promovem saúde ou perda de peso e em longo prazo provocam diversas consequências clínicas, físicas, emocionais e psicológicas, além do reganho do peso, na maioria das pessoas. (Alvarenga et al. 2015)

As estratégias têm como objetivos reduzirem os fatores de risco das Doenças Crônicas Não Transmissíveis (DCNTs), além de assegurar a qualidade da atenção aos pacientes, lidar com as questões sociais da saúde e defender os direitos das pessoas. Dentro dessa perspectiva, o Brasil ainda regulamentou leis federais para atenção às pessoas com obesidade no sistema de saúde pública, e os planos de saúde são obrigados a oferecer tratamento às pessoas com obesidade mórbida. (OPAS, 2015)

Nesse sentido, o controle deste problema requer a ampliação de estratégias compostas por profissionais de outros saberes, a fim de programar novas maneiras de combater o problema. Ratificando sobre a questão, o Ministério da Saúde recomenda o tratamento da psicoterapia, a fim de auxiliar no controle de peso e promover a motivação e habilidades para o enfrentamento e, consequentemente, o reganho de peso (ABESO, 2016 \& Ministério da Saúde, 2014).

A ciência do comportamento humano não tem como objeto de estudo a obesidade em si, mas a relação entre a pessoa e a obesidade e o contexto que ela está inserida, da qual se dá a partir da função que o comportamento alimentar tem para a pessoa. Por sua vez, a psicologia contribui com o aprimoramento da competência das pessoas em tomar decisões saudáveis para si mesmas e para o mundo, abandonar hábitos prejudiciais à saúde, ou, de maneira mais ampla, viver melhor. (Cavalcante, 2009).

Os estudos apontam que as avaliações psicológicas juntamente com instrumentos psicológicos, contribuem, não só para coletar dados e informações acerca das demandas e queixas dos pacientes, mas também os riscos de prosseguir com o tratamento da obesidade e prováveis desistências. (Marek et al., 2016).

Em 2013, o Conselho Federal de Psicologia, juntamente com os conselhos regionais lançaram uma "Cartilha de Avaliação Psicológica", a qual afirma que avaliação psicológica é um processo amplo de investigação, onde a pessoa expõe suas demandas, com o objetivo de proporcionar informações ao psicólogo para o levantamento de estratégias de enfrentamento, por meio de um conjunto de métodos reconhecidos pela psicologia (Conselho Federal de Psicologia, 2013).

Cohen et al. (2014) diferenciam avaliação psicológica da testagem psicologia. A primeira entende-se como uma coleta e integração dos dados que são identificados através de testes, entrevistas, estudos de caso, observação comportamental e procedimentos. Já a testagem psicológica os autores explicam como um processo de medição das variáveis, através de instrumentos ou procedimentos para obter um resultado ou amostra do comportamento.

A partir do entendimento do perfil epidemiológico possuir demandas específicas, o Ministério da Saúde (2018) elaborou a Agenda de Prioridades de Pesquisa do Ministério da Saúde (APPPMS), com o objetivo de identificar pesquisas que possam solucionar problemas de saúde pública, promover qualidade da atenção e desenvolver políticas públicas efetivas.

Portanto, quais os instrumentos utilizados na avaliação psicológica da pessoa obesa, no Brasil e no mundo? Esses instrumentos apontam e avaliam o que? 
Esta revisão pretende contribuir para o aprofundamento e sistematização do conhecimento do tema, assim como para uma análise do que tem sido produzido no Brasil e no mundo em relação aos instrumentos utilizados na avaliação psicológica da pessoa obesa. Assim, o objetivo desse estudo é analisar a literatura científica sobre instrumentos utilizados na avaliação psicológica da pessoa obesa.

\section{Metodologia}

Foi realizada uma revisão integrativa da literatura nacional e internacional, entre maio e junho de 2020. O estudo cumpriu seis etapas: I. Seleção da questão de pesquisa; II. Estabelecimento de critérios de inclusão e exclusão/ busca na literatura; III. Categorização dos estudos; IV. Avaliação dos estudos incluídos; V. Interpretação dos resultados; VI. Relato da revisão. (Mendes et al. 2008). O estudo visou à seguinte questão: Quais instrumentos psicológicos utilizados na avaliação psicológica no tratamento da obesidade?

A busca e a seleção dos estudos foram consultadas nas bases de dados: Literatura Latino-Americana e do Caribe em Ciências da Saúde (LILACS) e Index Psicologia - periódicos técnico-científicos via Biblioteca Virtual em Saúde (BVS), Medical Literature Analysis and Retrieval System Online (MEDLINE), via PubMed, e Scopus, via Portal de Periódicos Capes. Todo esse processo de pesquisa iniciou-se com consulta aos descritores em Ciências da Saúde (DeCS), por meio da BVS, ao Medical Subject Headings (MeSH), da National Library. Foram utilizados os seguintes descritores controlados: "psychometrics", "psychological test", "Psychological Assessment", "psychologist", "obesity", "overweight" e o descritor não controlado "psychological assessment", com os operadores booleanos "OR" $e$ "AND". Algumas variações na busca dos artigos foram realizadas, conforme o Quadro 1.

Quadro 1. Estratégias de busca nas bases de dados.

\begin{tabular}{|c|c|}
\hline Bases de Dados & Estratégia de Busca \\
\hline $\begin{array}{l}\text { LILACS e Index } \\
\text { Psicologia- periódicos } \\
\text { técnico-científicos } \\
\text { /BVS }\end{array}$ & $\begin{array}{l}\text { (tw:("Psychological Evaluation" OR "Psychological Diagnosis" OR "Psychological Outcomes" OR } \\
\text { "Psychological Assessment" OR Psychometric* OR "Psychological Test" OR "AvaliacaoPsicologica" OR } \\
\text { "TestePsicologico" OR Psicometria* OR "DesfechosPsicologicos" OR "DiagnosticoPsicologico" OR } \\
\text { "EvaluacionPsicologica" OR "PruebaPsicologica" OR "ResultadosPsicologicos")) AND (tw:(obesity OR } \\
\text { obesidad* OR overweight OR sobrepeso)) }\end{array}$ \\
\hline $\begin{array}{r}\mathrm{ME} \\
\mathbf{P u}\end{array}$ & 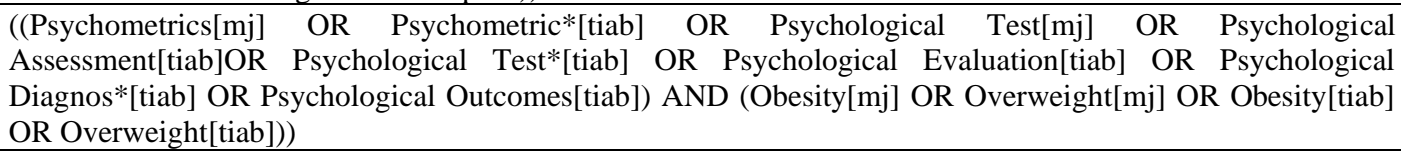 \\
\hline $\mathbf{S}$ & $\begin{array}{l}\text { (TITLE-ABS-KEY("Psychological Evaluation" OR "Psychological Diagnosis" OR "Psychological Outcomes" } \\
\text { OR "Psychological Assessment" OR Psychometric* OR "Psychological Test") AND TITLE-ABS- } \\
\text { KEY(Obesity OR Overweight)) }\end{array}$ \\
\hline
\end{tabular}

Fonte: Autores (2020).

Foram utilizados o recorte temporal dos últimos 10 anos e os idiomas português, inglês e espanhol. Como critérios de inclusão, os estudos deveriam apresentar artigos disponíveis na íntegra, público adulto, avaliação do psicólogo no tratamento da obesidade. Como critérios de exclusão foram estabelecidos as revisões, livros, monografias, resenhas, editoriais, teses, dissertações, temas que abordassem anorexia e bulimia, crianças e adolescentes.

Durante a busca nas bases de dados citadas foram identificados 963 artigos, dos quais 178 duplicados, 755 excluídos pelo título e resumo, 16 estudos excluídos por não responderem à questão norteadora e o objetivo do estudo que tratavam de temática, tais como: anorexia nervosa, bulimia, diabetes, depressão, avaliação nutricional, psiquiatra, e outras doenças clínicas, resultando, portanto, 14 estudos para análise. (Imagem 1) 
Cada artigo foi lido e revisado de forma minuciosa para levantar informações relevantes referentes à caracterização dos instrumentos psicológicos, e sua relevância e fidedignidade na avaliação psicológica no tratamento da obesidade.

Imagem 1 - Fluxograma de busca e seleção dos artigos.

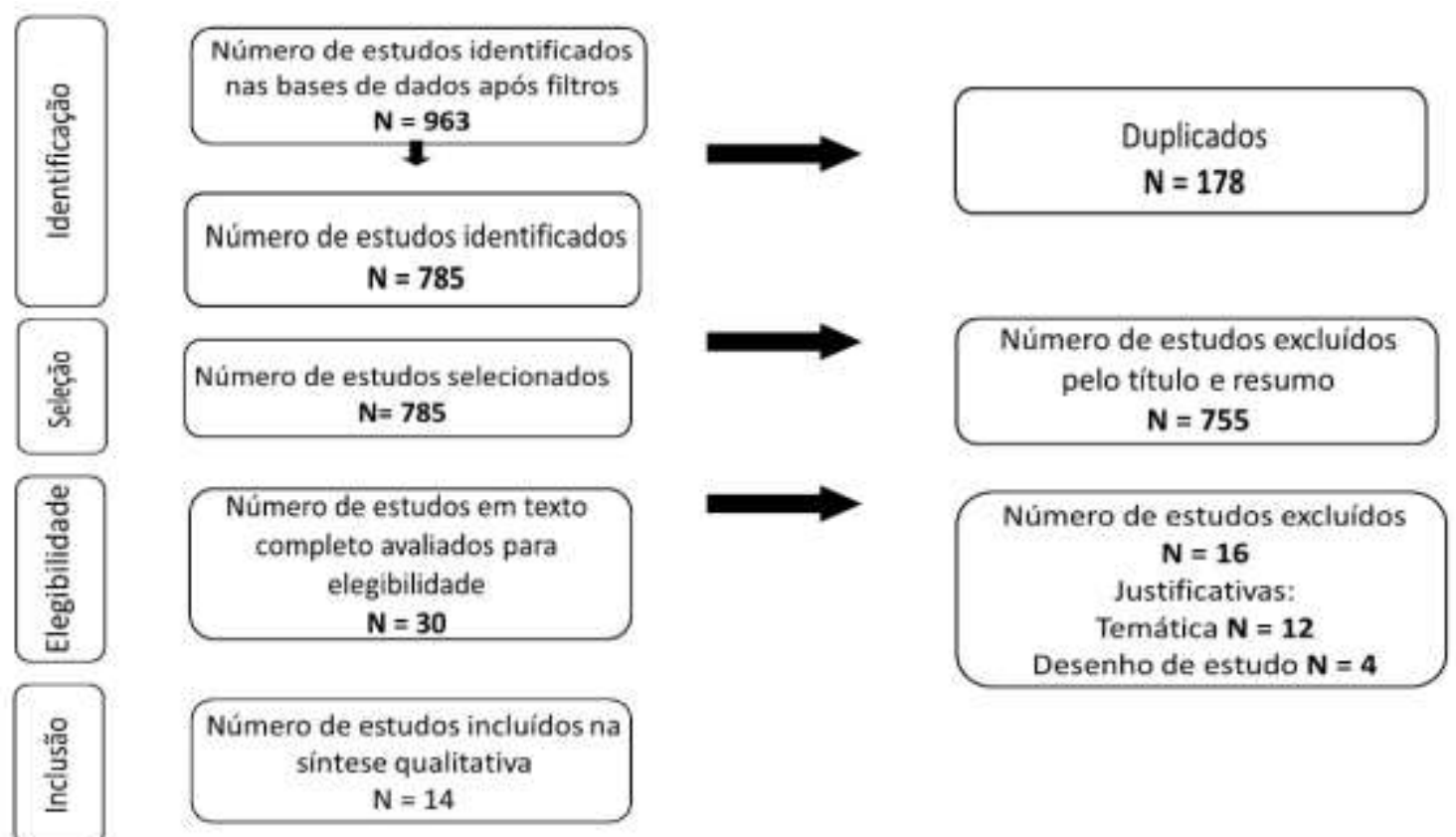

Fonte: Autores (2020).

\section{Resultados}

Para categorização dos estudos optou por elaborar as seguintes informações: autores, ano de publicação, país, objetivos do estudo, instrumentos utilizados e principais resultados, como consta no Quadro 2.

Quadro 2 - Características das avaliações psicológicas no tratamento da obesidade.

\begin{tabular}{|c|c|c|c|}
\hline $\begin{array}{l}\text { Autores/ } \\
\text { ano/País }\end{array}$ & Objetivos & Instrumentos utilizados & Principais resultados \\
\hline $\begin{array}{l}\mathrm{E}^{1} \text { Marek } \\
\text { et. al., } \\
(2016) \\
\text { EUA }\end{array}$ & $\begin{array}{c}\text { Observar quais pacientes } \\
\text { desistiriam de fazer a cirurgia no } \\
\text { prazo de um ano }\end{array}$ & $\begin{array}{c}\text { - Inventário Multifásico de Personalidade de } \\
\text { Minnesota-2 Escala MMPI-2-RF }\end{array}$ & $\begin{array}{l}\text { O uso de medidas na avaliação psicológica pode } \\
\text { atender a necessidade do paciente aderir ao } \\
\text { tratamento da obesidade. }\end{array}$ \\
\hline $\begin{array}{c}\mathrm{E}^{2} \\
\text { McLean } \\
\text { et. al., } \\
\text { (2016) } \\
\text { Reino } \\
\text { Unido }\end{array}$ & $\begin{array}{c}\text { Investigar o efeito da ansiedade e } \\
\text { depressão, usando diferentes } \\
\text { definições de critérios de } \\
\text { diagnóstico. }\end{array}$ & $\begin{array}{l}\text {-Escala Hospitalar de Ansiedade e - Depressão } \\
\text { (HADS) }\end{array}$ & $\begin{array}{l}\text { Pacientes com obesidade complexa que obtiveram } \\
\text { pontuação para sintomas graves de ansiedade e/ou } \\
\text { depressão e receberam apoio psicológico durante seu } \\
\text { programa de controle de peso obtiveram resultados } \\
\text { semelhantes de perda de peso de casos não graves. }\end{array}$ \\
\hline $\begin{array}{l}\mathrm{E}^{3} \text { Grothe } \\
\text { et. al., } \\
\text { (2014) } \\
\text { EUA }\end{array}$ & $\begin{array}{l}\text { Descrever os sintomas bipolares e } \\
\text { sua relação com fatores } \\
\text { psicológicos em uma amostra de } \\
\text { adultos que procuravam cirurgia } \\
\text { bariátrica }\end{array}$ & $\begin{array}{l}\text {-MDQ - Questionário de Transtorno do } \\
\text { Humor. } \\
\text {-Inventário de Depressão Beck II (BDI-II) } \\
\text {-Transtorno de Ansiedade Generalizada-7 } \\
\text { (GAD-7). } \\
\text {-Teste de identificação de transtornos por uso } \\
\text { de álcool (AUDITORIA) } \\
\text {-Questionário emocional de comer demais } \\
\text { (EOQ). } \\
\text {-Questionário de Trauma na Infância } \\
\text { (CTQ) } \\
\text {-Escala de tolerância à angústia (DTS) }\end{array}$ & $\begin{array}{c}\text { Sintomas bipolares são predominantes e podem } \\
\text { apresentar desafios na organização dos esforços para } \\
\text { a cirurgia bariátrica. }\end{array}$ \\
\hline
\end{tabular}


Research, Society and Development, v. 11, n. 1, e21111125038, 2022

(CC BY 4.0) | ISSN 2525-3409 | DOI: http://dx.doi.org/10.33448/rsd-v11i1.25038

\begin{tabular}{|c|c|c|c|}
\hline & & $\begin{array}{l}\text {-Questionário de estilo de vida de eficácia de } \\
\text { peso (WEL) }\end{array}$ & \\
\hline $\begin{array}{l}\text { E }^{4} \text { Schutt } \\
\text { et. al., } \\
\text { (2015) } \\
\text { EUA }\end{array}$ & $\begin{array}{c}\text { Avaliar instrumentos de } \\
\text { desempenho semelhante em um } \\
\text { ambulatório de cirurgia bariátrica }\end{array}$ & $\begin{array}{c}\text {-Escala de Depressão de Beck BDI-II } \\
\text {-Questionário de Saúde do Paciente (PHQ) }\end{array}$ & $\begin{array}{l}\text { A avaliação dos sintomas depressivos em candidatos } \\
\text { a cirurgia bariátrica é importante para determinar sua } \\
\text { adequação à cirurgia ou a necessidade de intervenção } \\
\text { psicológica ou psiquiátrica. }\end{array}$ \\
\hline $\begin{array}{l}E^{5} \text { Hall } \\
\text { et. al., } \\
(2013) \\
\text { EUA }\end{array}$ & $\begin{array}{l}\text { Avaliar a validade estrutural do } \\
\text { BDI-II e examinar a } \\
\text { confiabilidade e } \\
\text { validade deste instrumento em } \\
\text { uma avaliação de cirurgia } \\
\text { bariátrica. }\end{array}$ & $\begin{array}{l}\text {-PAI Personality Assessment Inventory } \\
\text {-Escala de Depressão de Beck - BDI-II }\end{array}$ & $\begin{array}{l}\text { O BDI é uma medida de avaliação importante para } \\
\text { melhorar resultado em candidatos que procuram } \\
\text { emagrecer através da cirurgia bariátrica. }\end{array}$ \\
\hline $\begin{array}{c}E^{6} \\
\text { RIBEIR } \\
\text { O et. al., } \\
\text { (2018) } \\
\text { Brasil }\end{array}$ & $\begin{array}{l}\text { Avaliar o perfil psicológico, antes } \\
\text { e após a cirurgia bariátrica }\end{array}$ & $\begin{array}{l}\text {-Inventário de Depressão de Beck (BDI) } \\
\text {-Ansiedade de Beck (BAI) } \\
\text {-Escala de Compulsão Alimentar (BES) }\end{array}$ & $\begin{array}{c}\text { Foi considerado importante a avaliação psicológica e } \\
\text { as intervenções apropriadas para pacientes } \\
\text { submetidos à cirurgia bariátrica, mesmo após a perda } \\
\text { de peso. }\end{array}$ \\
\hline $\begin{array}{l}E^{7} \text { Pataky } \\
\text { et. al., } \\
\text { (2018) } \\
\text { Suíça }\end{array}$ & $\begin{array}{l}\text { Avaliar os efeitos da perda de } \\
\text { peso sobre endocanabinóides, } \\
\text { cardiometabólicos e psicológicos, } \\
\text { bem como Qualidade de Vida } \\
\text { (QV) e elucidar o papel dos } \\
\text { endocanabinóides na síndrome } \\
\text { metabólica (EM). }\end{array}$ & $\begin{array}{c}\text {-Inventário de Depressão de Beck (BDI-II) } \\
\text {-Escala Hospitalar de Ansiedade e Depressão } \\
\text {-Versão francesa do Questionário de Exame } \\
\text { para Transtorno Alimentar (EDE-Q) } \\
\text {-Questionário de Alimentação de Três Fatores } \\
\text { (TFEQ) } \\
\text {-Impacto Weight on Quality of Life-Lite } \\
\text { (IWQoL-Lite) }\end{array}$ & $\begin{array}{l}\text { Além da perda de peso e melhora do perfil } \\
\text { cardiometabólico, o programa de perda de peso pode } \\
\text { ser benéfico em termos de resultados psicológicos, } \\
\text { distúrbios alimentares e melhoria da QV. Não houve } \\
\text { diferença na perda de peso entre os diferentes } \\
\text { métodos do controle de peso. }\end{array}$ \\
\hline $\begin{array}{l}\mathrm{E}^{8} \text { Hood } \\
\text { et. al., } \\
(2012) \\
\text { EUA }\end{array}$ & $\begin{array}{l}\text { Relatar à confiabilidade do BES } \\
\text { para pacientes que procuram } \\
\text { cirurgia bariátrica. }\end{array}$ & -Binge Eating Scale - BES & $\begin{array}{c}\text { Compulsão alimentar não afeta negativamente os } \\
\text { resultados da perda de peso em um ano após a } \\
\text { cirurgia. }\end{array}$ \\
\hline $\begin{array}{l}\mathrm{E}^{9} \text { Iceta } \\
\text { et. al., } \\
(2016) \\
\text { França }\end{array}$ & $\begin{array}{c}\text { Determinar se fatores } \\
\text { psicológicos pré-operatórios, } \\
\text { transtornos de personalidade (DP) } \\
\text { e comportamentos alimentares de } \\
\text { candidatos à cirurgia revisional } \\
\text { diferem dos candidatos à cirurgia } \\
\text { bariátrica primária. }\end{array}$ & $\begin{array}{l}\text {-Escala de Dependência Alimentar de Yale - } \\
\text { YFAS } \\
\text {-Structured Clinical Interview SCID-II } \\
\text {-Inventário de depressão de Beck - BDI } \\
\text {-Escala Hospitalar de Ansiedade e Depressão - } \\
\text { HADS } \\
\text {-Escala de autoestima de Rosenberg - RSES } \\
\text {-Teste Investigatório Bulímico, Edimburgo - } \\
\text { BITE }\end{array}$ & $\begin{array}{l}\text { Candidatos à cirurgia bariátrica não diferiram dos } \\
\text { candidatos a um procedimento clínico em relação a } \\
\text { comportamentos alimentares e traços psicológicos. } \\
\text { No entanto, o estudo confirma a necessidade de uma } \\
\text { extensa avaliação antes da cirurgia bariátrica, } \\
\text { incluindo a busca por transtornos de personalidade. }\end{array}$ \\
\hline $\begin{array}{l}\mathrm{E}^{10} \\
\text { Ortega } \\
\text { et. al., } \\
(2012) \\
\text { Espanha }\end{array}$ & $\begin{array}{l}\text { Determinar as variáveis pré- } \\
\text { operatórias que identificam os } \\
\text { sintomas psicológicos, de } 6 \text { e } 12 \\
\text { meses após a cirurgia, para } \\
\text { melhorar o resultado clínico de } \\
\text { obesos mórbidos submetidos à } \\
\quad \text { cirurgia bariátrica. }\end{array}$ & $\begin{array}{l}\text {-Symptom Checklist-90-Revised (SCL-90-R } \\
\text {-Body Shape Questionnaire (BSQ) } \\
\text {-Rosenberg Self-Esteem Questionnaire } \\
\text {-Medical Outcomes Study } \\
\text {-Social Support Survey }\end{array}$ & $\begin{array}{l}\mathrm{O} \text { apoio instrumental e o enfrentamento das emoções } \\
\text { foram os melhores preditores de } 6 \text { meses de } \\
\text { sofrimento psíquico no pós-operatório; no entanto, } \\
\text { eles perderam seu valor preditivo ao longo do tempo. } \\
\text { O principal preditor de sintomas psicológicos, } 1 \text { ano } \\
\text { de pós-operatório permaneceu quase exclusivamente } \\
\text { a imagem corporal. }\end{array}$ \\
\hline $\begin{array}{l}\mathrm{E}^{11} \\
\text { Agüera } \\
\text { et. al., } \\
\text { (2015) } \\
\text { Espanha }\end{array}$ & $\begin{array}{l}\text { Identificar preditores clínicos, } \\
\text { psicopatológicos e de } \\
\text { personalidade que interfere no } \\
\text { sucesso do tratamento em } \\
\text { pacientes obesos graves. }\end{array}$ & $\begin{array}{l}\text {-Eating Disorder Inventory-2 (EDI-2) } \\
\text {-Symptom Checklist-90 Items-Revised (SCL- } \\
\text { 90-R) } \\
\text {-Temperament and Character Inventory- } \\
\text { Revised (TCI-R) } \\
\text {-Barratt Impulsiveness Scale-11 (BIS-11) }\end{array}$ & $\begin{array}{l}\text { Traços psicopatológicos e de personalidade podem } \\
\text { afetar a perda de peso após a cirurgia bariátrica e suas } \\
\text { comorbidades metabólicas associadas. }\end{array}$ \\
\hline $\begin{array}{l}\text { E }^{12} \text { Ahnis } \\
\text { et. al., } \\
(2012) \\
\text { Alemanh } \\
\quad \text { a }\end{array}$ & $\begin{array}{l}\text { Identificar as variáveis que } \\
\text { preveem a descontinuação } \\
\text { prematura do tratamento da } \\
\text { obesidade }\end{array}$ & $\begin{array}{c}\text {-SOZ - Questionário sobre características } \\
\text { sociais (medida em alemão usada internamente } \\
\text { pelo hospita } \\
\text {-VEF - Fragebogen zum Essverhalten } \\
\text { (questionário sobre comportamento alimentar; } \\
\text { original em alemão } \\
\text {-EDI } 2 \text { - inventário de transtornos alimentares } \\
\text {-PSQ-20 - questionário de estresse percebido } \\
\text {-GBB-24 - Giessener Beschwerdebogen } 34 \\
\text { ( Lista de reclamações subjetivas de Giessen } \\
\text {-ISR - CID-10-classificação de sintomas } \\
\text {-BSF - Berliner Stimmungsfragebogen } \\
\text { ( Questionário de humor de Berlim) } \\
\text {-SF-8 - German version of the health survey } \\
\text {-Escala de depressão do PhQ - questionário de } \\
\text { saúde do paciente (versão alemã: PhQ }\end{array}$ & $\begin{array}{l}\text { Os resultados demonstraram que o programa utilizado } \\
\text { para perda de peso deve ser adaptado para obter } \\
\text { melhores resultados na persistência dos participantes, } \\
\text { e prevenir a desistência do tratamento. }\end{array}$ \\
\hline
\end{tabular}




\begin{tabular}{|c|c|c|c|}
\hline & & $\begin{array}{l}\text {-SWOP - Fragebogen zu Selbstwirksamkeit, } \\
\text { Optimismus und Pessimismus -SOC-9 - } \\
\text { Versão alemã da escala de senso de coerência } \\
\text { de Antonovsky }\end{array}$ & \\
\hline $\begin{array}{l}\mathrm{E}^{13} \\
\text { Mahony } \\
\text { et. al., } \\
(2010) \\
\text { EUA }\end{array}$ & $\begin{array}{l}\text { Determinar a confiabilidade e a } \\
\text { validade de construção do } \\
\text { PsyBari. }\end{array}$ & $\begin{array}{c}\text {-PsyBari Subscales } \\
\text {-Faking Good/Minimization/Denial Scale } \\
\text {-Surgical Motivation Scale } \\
\text {-Emotional Eating Scale } \\
\text {-The Anger Scale } \\
\text {-Obesity-Related Depression Scale } \\
\text { Anecdotally } \\
\text {-Weight-Related Impairment Scale } \\
\text {-Weight-Related Social Impairment Scale } \\
\text {-Knowledge of Postsurgical Dietary } \\
\text { Restrictions Scale } \\
\text {-Substance/Alcohol Abuse Scale } \\
\text {-Surgical Anxiety Scale }\end{array}$ & $\begin{array}{l}\text { Os resultados indicam que existem parâmetros } \\
\text { psicométricos únicos na construção de testes para } \\
\text { pacientes em cirurgia bariátrica. O PsyBari tem boa } \\
\text { confiabilidade geral, embora duas das } 11 \text { subescalas } \\
\text { tenham pouca confiabilidade. }\end{array}$ \\
\hline $\begin{array}{l}\mathrm{E}^{14} \text { Fox } \\
\text { et. al., } \\
(2015) \\
\text { Reino } \\
\text { Unido }\end{array}$ & $\begin{array}{c}\text { Identificar as emoções, supressão } \\
\text { emocional e redução da } \\
\text { expressividade emocional na } \\
\text { previsão de comportamentos } \\
\text { compulsivos alimentares em } \\
\text { pessoas obesas. }\end{array}$ & $\begin{array}{c}\text {-Perception of Threats from Emotion - } \\
\text {-Beck Depression Inventory -BDI } \\
\text {-Emotional Expressiveness Scale } \\
\text {-Dutch Eating Behavior Questionnaire } \\
\text { (DEBQ) } \\
\text {-Binge Eating Scale }\end{array}$ & $\begin{array}{l}\text { Os resultados deste estudo destacam a importância de } \\
\text { entender a emoção de pessoas que comem } \\
\text { compulsivamente com objetivo de suprimir suas } \\
\text { emoções. }\end{array}$ \\
\hline
\end{tabular}

Fonte: Autores (2020).

O número de publicações de maior concentração foi entre os anos de 2012 (n=3), 2015 (n=3) e 2016 (n=3). O país com maior número de estudos foram os Estados Unidos ( $n=6)$, seguido dos países europeus: Espanha ( $n=2)$, Reino Unido ( $n=2)$, Alemanha (n=1), França (n=1), Suíça (n=1), e América do Sul: Brasil (n=1).

Nos últimos anos, tem-se observado um aumento no número de cirurgias bariátricas realizadas no Brasil e no mundo (Siqueira e Zanotti, 2017), e podemos constatar esses resultados nesta pesquisa, pois, o método cirúrgico foi o tratamento da obesidade mais encontrado ( $n=11)$, seguido por programas clínicos interdisciplinares $(n=2)$ e o estudo com tratamento clínico ou cirúrgico $(\mathrm{n}=1)$.

Três estudos, como consta no Quadro 2, utilizaram um instrumento para avaliação psicológica, enquanto nos demais, estudos variados; destacando a pesquisa de Ahnis et. al. (2012) que levantou 11 instrumentos de avaliação.

Os instrumentos mais utilizados nos programas de perda de peso foram respectivamente o Beck Depression Inventory - BDI (Inventário de Depressão de Beck) (n=7); o Binge Eating Scale - BES (Escala de Compulsão Alimentar Periódica - TCAP) (n=3); e a Escala Hospitalar de Ansiedade e Depressão - HADS (n=3).

Outro dado importante do presente estudo, é a prevalência de mulheres nos serviços clínicos e cirúrgicos para o tratamento da obesidade. Essa constatação confirma estudos anteriores, como o de Mahony (2010), que afirmam que mulheres procuram com mais regularidade os serviços de atendimento a cirurgia bariátrica e controle de peso.

Os instrumentos utilizados na avaliação psicológica da obesidade tinham como temas dominantes, identificar sintomas como depressão, ansiedade e compulsão alimentar. No quadro 3, destacamos os demais temas encontrados nos estudos no tratamento da obesidade. 
Quadro 3 -Temas relevantes encontrados nas avaliações psicológicas.

Abuso físico, sexual e emocional na infância
Autoconhecimento
Autoeficácia alimentar
Autoestima
Bulimia
Busca pela magreza
Capacidade de reconhecer e expressar emoções
Capacidade de suportar estados psicológicos negativos
Comprometimento com o tratamento
Comprometimento físico, recreativo e ocupacional devido ao peso.
Frustração
Impulsividade
Irritabilidade
Nível de atividade social.
Percepção de sentimentos de fome
Perfeccionismo
Preocupação Alimentar
Preocupação com a forma do corpo ou a satisfação da forma corporal
Raiva
Regulação de impulsos
Restrição alimentar e fome.
Sintomas depressivos devido ao peso
Sintomas psiquiátricos

Fonte: Autores (2020).

O presente estudo demonstrou em seus resultados que os instrumentos mais utilizados no tratamento da obesidade são internacionais (América do Norte e Europa), mesmo no estudo de Ribeiro et. al., (2018) realizado no Brasil, foram utilizados instrumentos estrangeiros. No total, foram identificados 52 instrumentos, entre escalas, testes e questionários, que são listados no Quadro 4.

Quadro 4. Instrumentos utilizados no tratamento da obesidade.

\begin{tabular}{|l|l|}
\hline \multicolumn{1}{|c|}{ Instrumento, escalas, testes e questionários } & \multicolumn{1}{c|}{ Objetivo } \\
\hline 1. Escala MMPI-2-RF & $\begin{array}{l}\text { Avaliar a psicopatologia em 5 domínios: funcionamento emocional, pensamento, } \\
\text { comportamental, somático / cognitivo e interpessoal. }\end{array}$ \\
\hline 2. Escala Hospitalar de Ansiedade e Depressão & Detectar estados de depressão e ansiedade no ambiente de um ambulatório médico hospitalar. \\
\hline 3. Mood Disorder Questionnaire-MDQ & Avaliar sintomas bipolares. \\
\hline 4. Beck Depression Inventory - BDI-II & Avaliar sintomas de depressão. \\
\hline 5. Generalized Anxiety Disorder-7 & Avalia sintomas de Ansiedade Generalizada (GAD -7). \\
\hline $\begin{array}{l}\text { 6. On The Alcohol Use Disorders Identification Test } \\
\text { (AUDITORIA) }\end{array}$ & Avalia transtornos por uso de substâncias e álcool. \\
\hline 7. Childhood Trauma Questionnaire - CTQ & Avalia abuso e negligência física, sexual e emocional na infância. \\
\hline $\begin{array}{l}\text { 8. Distress Tolerance Scale - DTS (Escala de Tolerância } \\
\text { a Angustia) }\end{array}$ & Avalia a capacidade de suportar estados psicológicos negativos. \\
\hline 9. Weight Efficacy Lifestyle Questionnaire (WEL) & Questionário de estilo de vida que avalia autoeficácia alimentar. \\
\hline 10. Patiente Health Questionnaire (PHQ-9) & Derivado do PRIME-MD, tem como objetivo avaliar a depressão na atenção primária. \\
\hline 11. Personality Assessment Inventory (PAI) & Avaliar sintomas afetivos, cognitivos e somáticos da depressão. \\
\hline 12. Binge Eating Scale - BES & Avaliar a presença de sintomas de compulsão alimentar. \\
\hline $\begin{array}{l}\text { 13. Versão francesa do Questionário de Exame para } \\
\text { Transtorno Alimentar (EDE-Q) }\end{array}$ & $\begin{array}{l}\text { Abrange quatro subescalas (Restrição, Preocupação alimentar, Preocupação de forma e } \\
\text { preocupação de peso). }\end{array}$ \\
\hline 14. Questionário de Alimentação de Três Fatores (TFEQ) & Medir dimensões, como: restrição cognitiva da alimentação, desinibição e fome. \\
\hline 15. Impacto Weight on Quality of Life-Lite - IWQol-Lite & Objetivos avaliar a vida social, profissional, sexual, autoestima e mobilidade. \\
\hline 16. Binge Eating Scale (BES) & Avaliar a presença de sintomas de compulsão alimentar. \\
\hline 17. Structured Clinical Interview SCID-II & Diagnósticos padronizados dos transtornos de Personalidade. \\
\hline
\end{tabular}


Research, Society and Development, v. 11, n. 1, e21111125038, 2022

(CC BY 4.0) | ISSN 2525-3409 | DOI: http://dx.doi.org/10.33448/rsd-v11i1.25038

\begin{tabular}{|c|c|}
\hline 18. Escala de autoestima de Rosenberg - RSES & Medir a autoestima. \\
\hline 19. Teste Investigatório Bulímico, Edimburgo - BITE & $\begin{array}{l}\text { É uma medida de autorrelato para avaliar a gravidade e a frequência dos sintomas da compulsão } \\
\text { alimentar. }\end{array}$ \\
\hline 20. Escala de Dependência Alimentar de Yale - YFAS & Medir os sintomas de dependência alimentar que ocorreram durante os últimos 12 meses. \\
\hline 21. Symptom Checklist-90-Revised (SCL-90-R) & Avaliar sintomas psiquiátricos em pacientes ambulatoriais. \\
\hline 22. Body Shape Questionnaire (BSQ & Avaliar a satisfação da forma corporal. \\
\hline 23. Rosenberg Self-Esteem Questionnaire & Escalas utilizada para avaliação da autoestima. \\
\hline 24. Medical Outcomes Study Social Support Survey & Avaliar diversas dimensões da vida. \\
\hline 25. Eating Disorder Inventory-2 (EDI-2) & $\begin{array}{l}\text { O EDI-2 mantém os } 64 \text { itens agrupados em } 11 \text { escalas: busca pela magreza, insatisfação } \\
\text { corporal, bulimia, ineficácia, perfeccionismo, desconfiança interpessoal, consciência } \\
\text { interoceptiva, medos de maturidade, ascetismo, regulação de impulsos e insegurança social. }\end{array}$ \\
\hline 26. Symptom Checklist-90 (SCL-90-R) & Avaliar sofrimento psíquico e psicopatologia autorreferidos. \\
\hline $\begin{array}{l}\text { 27. Temperament and Character Inventory-Revised (TCI- } \\
\text { R) }\end{array}$ & Avaliar o temperamento. \\
\hline 28. Barratt Impulsiveness Scale-11 (BIS-11) & Avalia diferentes aspectos da impulsividade. \\
\hline 29. SOZ - Questionnaire on social characteristics & $\begin{array}{l}\text { Questionário sobre características sociais (medida em alemão usada internamente pelo } \\
\text { hospital). }\end{array}$ \\
\hline 30. VEF - Fragebogen zum Essverhalten & $\begin{array}{l}\text { Avalia o comportamento alimentar, agrupados em três escalas: "Controle cognitivo do } \\
\text { comportamento alimentar / controle / restrição da alimentação", "Perturbação do } \\
\text { comportamento alimentar" e "Percepção de sentimentos de fome". }\end{array}$ \\
\hline 31. EDI 2 - eating disorder inventory & $\begin{array}{l}\text { Avalia psicopatologias específicas de pacientes com anorexia e bulimia nervosa e outros } \\
\text { transtornos alimentares psicogênicos. }\end{array}$ \\
\hline 32. PSQ-20 - perceived stress questionnaire & $\begin{array}{l}\text { Avalia as atuais percepções subjetivas de estresse, resumidos em quatro escalas intituladas } \\
\text { "Preocupações", "Tensão", "Alegria" e "Demandas". }\end{array}$ \\
\hline 33. GBB-24 - Giessener Beschwerdebogen & $\begin{array}{l}\text { Avaliando vários complexos de queixas subdivididos em quatro escalas: "Exaustão", } \\
\text { "Desconforto abdominal superior", "Articulações e músculos doloridos", "Queixas cardíacas } \\
\text { subjetivas" e o escore total da escala "Pressão de queixas subjetivas. }\end{array}$ \\
\hline 34. ISR - CID-10-symptom-rating & $\begin{array}{l}\text { Avalia sintomas, listados em cinco escalas: "Síndrome depressiva", "Síndrome de ansiedade", } \\
\text { "Síndrome obsessiva", "Síndrome somatoforme", "Síndrome do distúrbio alimentar. }\end{array}$ \\
\hline $\begin{array}{l}\text { 35. SF - Berliner Stimmungsfragebogen (Questionário } \\
\text { de humor de Berlim) }\end{array}$ & $\begin{array}{l}\text { Avalia "Cansaço", "Apatia", "Depressão ansiosa", "Raiva", "Comprometimento", "Bom } \\
\text { humor. }\end{array}$ \\
\hline $\begin{array}{l}\text { 36. Depression scale of the PHQ - patient health } \\
\text { questionnaire PHQ }\end{array}$ & Avalia sintomas de depressão. \\
\hline 37. SF-8 - German version of the health survey & $\begin{array}{l}\text { Avalia a qualidade de vida relacionada à saúde, usando os dois escores totais de "saúde } \\
\text { mental" e "saúde física. }\end{array}$ \\
\hline $\begin{array}{l}\text { 38. SWOP - Fragebogen zu Selbstwirksamkeit, } \\
\text { Optimismus und Pessimismus } 40\end{array}$ & Avaliação de crenças em autoeficácia e otimismo. \\
\hline $\begin{array}{l}\text { 39. SOC-9 - German version of Antonovsky's sense of } \\
\text { coherence scale (formerly the orientation to life scale. }\end{array}$ & $\begin{array}{l}\text { Versão alemã da escala de senso de coerência de Antonovsky (anteriormente a escala de } \\
\text { orientação para a vida). }\end{array}$ \\
\hline 40. PsyBari Subscales & Avaliações psicológicas pré-cirúrgicas. \\
\hline 41. Faking Good/Minimization/Denial Scale & $\begin{array}{l}\text { Avalia a consciência e vontade de reconhecer comportamentos comumente relatados e /ou } \\
\text { estados psicológicos relacionados à obesidade, relacionado à sua motivação em continuar o } \\
\text { tratamento. }\end{array}$ \\
\hline 42. Surgical Motivation Scale & $\begin{array}{l}\text { A Escala da Raiva avalia a consciência e a vontade de reconhecer e gerenciar a frustração, } \\
\text { irritabilidade e raiva. }\end{array}$ \\
\hline 43. Emotional Eating Scale & Avalia a compulsão alimentar. \\
\hline 44.The Anger Scale & Escala que mede apenas sintomas depressivos devido ao peso. \\
\hline 45. Obesity-Related Depression Scale Anecdotally & Mede o comprometimento físico, recreativo e ocupacional devido ao peso. \\
\hline 46. Weight-Related Impairment Scale & $\begin{array}{l}\text { A Escala de Comprometimento Social Relacionado ao Peso avalia quanto o peso prejudica } \\
\text { seu nível de atividade social. }\end{array}$ \\
\hline 47. Weight-Related Social Impairment Scale & Essa escala avalia o conhecimento das mudanças pós cirúrgicas. \\
\hline 48. Knowledge of Postsurgical Dietary Restrictions Scale & Avaliar o uso de substancias e álcool. \\
\hline $\begin{array}{l}\text { 49. Substance/Alcohol Abuse Scale } \\
\text { Surgical Anxiety Scale }\end{array}$ & Avalia a ameaça representada por emoções como tristeza, felicidade, raiva, medo e nojo. \\
\hline 50. Perception of Threats from Emotion & Avalia a capacidade de reconhecer e expressar emoções. \\
\hline 51. Emotional Expressiveness Scale & Mede a restrição alimentar, externa e emocional. \\
\hline 52. Dutch Eating Behavior Questionnaire (DEBQ) & Medição o comer restrito, externo e emocional. \\
\hline
\end{tabular}

Fonte: Autores (2020). 


\section{Discussão}

Os resultados dessa pesquisa apresentaram diversos instrumentos, escalas, questionários e testes psicológicos que tem como objetivo diagnosticar transtornos alimentares ou mentais que interferem na obesidade e os impactos que a obesidade promove nas pessoas. Porém, constatou-se uma ausência de instrumentos de avaliação psicológica para o tratamento da obesidade.

Visto que, dentro de uma amostra de pessoas obesas que procuravam tratamento para a obesidade, os comportamentos compulsivos referiam-se a supressão emocional. Ou seja, não são todas as pessoas obesas que se encaixam em uma categoria diagnóstica. A alimentação emocional refere-se ao impulso de comer como uma reação aos sentimentos negativos ou estresse. Diante de emoções de tristeza e medo, por exemplo, o estudo Fox et al. (2015) apontou que níveis de déficit de habilidade de enfrentamento previam comportamentos de compulsão alimentar.

Como observamos, os instrumentos mais utilizados nos programas de perda de peso foram respectivamente o Beck Depression Inventory - BDI (Inventário de Depressão de Beck); o Binge Eating Scale - BES (Escala de Compulsão Alimentar Periódica - TCAP); e a Escala Hospitalar de Ansiedade e Depressão - HADS. Emoções como ansiedade, estresse e depressão podem ser uma das principais causas para a predispor a fome emocional. (Litwin; Goldbacher; Cardaciotto \& Gambrel, 2016)

As emoções afetam a alimentação de várias maneiras, uma das quais é comer para regula-las. Isso sugere que alguns indivíduos podem estar em maior risco de adotar práticas alimentares não saudáveis, e isso pode ser parte de uma adoção mais ampla de estratégias de enfrentamento mal-adaptativas em contextos diversos. (Safer et al., 2020)

Sobre essa perspectiva, o instrumento que mais se diferenciou dos demais foi o estudo de Marek et. al., (2016) Ahnis et. al., (2012) que enfatizaram o comprometimento em prosseguir com o tratamento da obesidade. Esses autores identificaram os fatores que influenciam as pessoas a desistirem durante o processo de avaliação, como: antissocial, ansiedade e abuso de substâncias. Eles destacaram a importância do uso de instrumentos na avaliação psicológica para identificar transtornos psicológicos, mas, também, fatores que impedem a pessoa prosseguir com o tratamento, destacando o uso de uma medida psicológica validada com essa população para fornecer feedback aos pacientes sobre sua saúde mental e fatores de impedimento ao tratamento.

É relevante destacar porque o psicólogo na avaliação inicial e durante o tratamento da obesidade contribuem para que os pacientes tenham maior engajamento e resultados satisfatórios. Sendo assim, um programa multidisciplinar que envolva a atuação do psicólogo no processo de avaliação, comprometimento e desenvolvimento das habilidades emocionais, traz benefícios a saúde, melhoria na qualidade de vida e, consequentemente, a perda de peso. (Pataky et al., 2018)

As avaliações psicológicas contribuem, não só para coletar dados e informações acerca das demandas e queixas dos pacientes, mas também tem como objetivo identificar os riscos de prosseguir com o tratamento e prováveis desistências. Por isso, que a participação do psicólogo nesse processo aumenta o engajamento no tratamento e obter maiores resultados na qualidade de vida (Lean, Morrison, Shearer, Boyle \& Logue, 2016). Ainda mais se o tratamento da obesidade for implementado com uma equipe multidisciplinar, composta por enfermeiros, médicos, psicólogos e nutricionistas, especificamente, obesidade grau III. (Nicolau et al. 2017)

Cabe ressaltar que a obesidade foi incluída no Manual Diagnóstico e Estatístico de Transtornos Mentais DSM-V (Diagnostic and Statistical Manual of Mental Disorders) como uma condição clínica, mas não categorizada como um transtorno mental. A doença envolve fatores comportamentais e emocionais relacionadas à alimentação, mas pode estar associada a transtornos psicológicos como depressão, ansiedade, alimentar, distorção da imagem ou pessoas que não entram em uma categoria diagnostica, como casos de baixa autoestima e comer emocional. (APA, 2014).

Outro fator determinante encontrado dentro dos critérios estabelecidos é a pouca produção Brasileira de avaliação referente a essa temática, em comparação com as contribuições dos estudos internacionais. A diferença é expressiva, visto que 
1 (um) estudo brasileiro foi selecionado, frente a 13 estudos internacionais. Mesmo com uma busca minuciosa sobre os achados, não encontramos um instrumento que pelo menos se aproximasse desta característica. É um dado importante a ser enfatizado, para que novos estudos sejam produzidos no Brasil com essa temática.

Avaliações psicológicas são recomendadas antes que os pacientes prossigam com a cirurgia bariátrica ou programas de controle de peso, tanto para identificar fatores que podem atrapalhar os resultados e o aprimoramento dos recursos a serem implementados no processo. (Oliveira et al. 2018)

Nossos resultados apontaram a prevalência de mulheres procurando os serviços de emagrecimento, que é um fator importante a ser considerado. Esse público foi predominante no tratamento clínico ou cirúrgico da obesidade. A hipótese sobre essa questão é porque há uma preocupação maior com a saúde e ideal de beleza, ambos associados à magreza das demandas sociais que incidem sobre as mulheres. Elas procuram esses serviços especializados, porque têm uma preocupação maior com a saúde e o corpo, ambos em resposta a uma demanda social. (Ribeiro et.al., 2018)

Esses dados apontam uma atenção especial porque, mesmo com o ideal de beleza associados à magreza exigida às mulheres, outros fatores podem contribuir para essa procura. O estudo apresentado por Pazooki et. al. (2014), indicou que mulheres obesas obtiveram maior pontuação em padrões emocionais de privação, desconfiança, fracasso, dependência, autossacrifício, especialmente abandono, isolamento social, esquemas de vulnerabilidade, autocontrole e subjugação. Os achados mostraram, também, que mulheres obesas sofriam de problemas cognitivos, vulnerabilidade emocional e comportamental em comparação com mulheres com peso saudável.

Outra categoria encontrada é a predominância do tratamento da obesidade através da cirurgia bariátrica. Os métodos tradicionais de avaliação incluem uma combinação de entrevistas clínicas, questionários, instrumentos, inventários, teste de personalidade / psicopatologia, testes de função cognitiva. (Dias et. Al., 2017).

Estudo de Ribeiro et al. (2018) comparou diferentes momentos após a cirurgia bariátrica e observou que a maior perda de peso acontece até os 23 primeiros meses após a cirurgia. Depois disso, o peso é estabilizado entre 23 e 59 meses. Após 60 meses, o peso aumenta gradualmente. Embora os pacientes apresentem bons resultados alguns meses depois da cirurgia, observase que manter o peso por toda a vida é o grande desafio que as pessoas obesas enfrentam e os profissionais envolvidos nesse processo. Considerando esses, é preciso elaborar de estratégias que promovam não só resultados da perda de peso, mas que promovam a manutenção do peso, ou seja, resultados consistentes e permanentes.

Poubel e Rodrigues (2018) acrescentam que o desenvolvimento de habilidades psicológicas o que favorece uma compreensão das limitações e potenciais que a história pessoal proporcionou. Os autores enfatizam a importância de estratégias cognitivas, comportamentais e emocionais, que tornam as pessoas capazes de influenciar o ambiente em que vivem, mesmo que tenham que se privar imediatamente de ganhos ou submeter-se a desconfortos para conquistar ganhos maiores ou evitar problemas em longo prazo

\section{Considerações Finais}

A promoção da saúde tem sido difundida como o bem mais precioso em todo o mundo. Além dos recursos médicos e nutricionais de prevenção e intervenção da obesidade, enfatizados em temas de livros, artigos e congressos, é necessário formular e aplicar estratégias psicológicas a fim de tornar sustentável o emagrecimento ou controle do peso, evitando a prevalência da doença e os custos que ela acarreta.

Mesmo com a crescente da obesidade no Brasil e no mundo, não foram identificados nas pesquisas levantadas na revisão integrativa, questionários, inventários, testes ou escalas específicas para o tratamento da obesidade, somente encontrado recursos com objetivo de identificar os impactos histórico-emocionais da pessoa obesa com a função de diagnosticar transtorno psicológico que interferisse no tratamento ou no processo de emagrecimento; mas não encontramos nenhum instrumento de 
avaliação que identificasse ou apontasse, não só os impactos sociais, emocionais, comportamentais, cognitivos, nutricionais e clínicos da pessoa obesa, mas instrumentos de avaliação que identificasse quais habilidades psicológicas esse público precisa desenvolver a fim de favorecer o tratamento e alcançar um novo estilo de vida.

A avaliação psicológica está entre uma das mais importantes atividades do psicólogo. Nesse aspecto, desenvolver materiais, a fim de favorecer a sociedade, é um dos compromissos da área de psicologia. Para tanto, o profissional de psicologia pode utilizar instrumentos para coletar informações sobre características comportamentais, cognitiva e emocionais da obesidade.

Portanto, abrimos a discussão sobre a importância de elaboração de métodos de avaliação, como instrumentos, escalas, testes ou questionários, que tenham como função diagnosticar as pessoas com transtornos mentais que interferem na obesidade, mas a importância de elaborar instrumentos que identifiquem as habilidades que a pessoa obesa precisaria desenvolver para emagrecer ou instalar hábitos alimentares saudáveis.

Estudos posteriores podem elaborar ferramentas específicas para avaliação psicológica à pessoa obesa, tanto em tratamento cirúrgico ou clínico, bem como na identificação das habilidades que essa população precisa desenvolver durante e pós-tratamento. Além de servir de subsídio para um atendimento sistematizado a esse público, sugerimos um instrumento que contribuirá com as intervenções e melhoria da qualidade do atendimento dos profissionais de saúde prestado ao obeso, articulado às ações da equipe multidisciplinar com foco na mudança na maneira de pensar, sentir e agir, a fim de promover a saúde e qualidade de vida.

Para avançar no processo da tecnologia e inovação do trabalho multiprofissional, na prevenção à obesidade e promoção da saúde no Brasil, propõe-se novos estudos que enfatizem as habilidades do obeso e não só na identificação dos transtornos mentais que a pessoa obesa adquiriu durante sua história, bem como, novas tecnologias que favorecerão a avaliação psicológica no tratamento da obesidade.

Com isso, espera-se intervir em um problema que emerge em todo o mundo, apoiando-se na premissa de que essa pesquisa contribuirá no atendimento direcionado ao atendimento ambulatorial e clínico a obesos e/ou pessoas que desejam, não só perder peso, mas manter hábitos alimentares saudáveis pós tratamento.

Sendo assim, orientamos, com base nos resultados deste trabalho, para que outros trabalho futuros, elaborem novas tecnológicas no processo de avaliação psicológica para cirurgia bariátrica e programas clínicos de controle ou perda de peso, a fim de favorecer um atendimento sistematizado a esse público, buscando assim, uniformidade nas intervenções e melhoria da qualidade do atendimento dos profissionais de saúde prestado ao obeso, articulado as ações da equipe multidisciplinar com foco na mudança de estilo de vida, visando à promoção da saúde e melhor qualidade de vida, não só na identificação dos transtornos mentais e seus impactos, mas na avaliação psicológica de pessoas obesas que não entram em uma categoria diagnosticas dos transtornos mentais.

\section{Referências}

Alvarenga, M., Figueiredo, M., Timerman, F., \& Antonaccio, C. (2015). Nutrição Comportamental. Manoele.

Agüera, Z., García-Ruiz-de-Gordejuela, A., Vilarrasa, N., Sanchez, I., Baño, M., Camacho, L., Granero, R., Jiménez-Murcia, S., Virgili, N., Lopez-Urdiales, R., de Bernabe, M. M.-G., Garrido, P., Monasterio, C., Steward, T., Pujol-Gebelli, J., Fernández-Aranda, F., \& Menchón, J. M. (2015). Psychological and Personality Predictors of Weight Loss and Comorbid Metabolic Changes After Bariatric Surgery. European Eating Disorders Review, $23(6)$, 509-516. https://doi.org/10.1002/erv.2404

Ahnis, A., Riedl, Figura, Steinhagen-Thiessen, \& Klapp. (2012). Psychological and sociodemographic predictors of premature discontinuation of a 1-year multimodal outpatient weight-reduction program: an attrition analysis. Patient Preference and Adherence, 165. https://doi.org/10.2147/ppa.s28022

Associação Brasileira para o Estudo da Obesidade e da Síndrome Metabólica. (2016). Rio de Janeiro: ANS. https://abeso.org.br/wpcontent/uploads/2019/12/Diretrizes-Download-Diretrizes-Brasileiras-de-Obesidade-2016.pdf

Agência Nacional de Saúde Suplementar. (2017). Manual de Diretrizes para o Enfretamento da Obesidade na Saúde Suplementar Brasileira. http://www.ans.gov.br/images/Manual_de_Diretrizes_para_o_Enfrentamento_da_Obesidade_na_Sa\%C3\%BAde_Suplementar_Brasileira.pdf 
Agenda de Prioridades de Pesquisa do Ministério da Saúde. (2018). Brasília: Ministério da Saúde. file:///C:/Users/vgs/Downloads/agenda_prioridades_pesquisa_ms\%20(2).pdf Conselho Federal de Psicologia. (2013). Cartilha Avaliação Psicológica. (1 ${ }^{\mathrm{a}} \quad$ Ed). Brasília: CFP. http://satepsi.cfp.org.br/docs/Avaliac\%CC\%A7aopsicologicaCartilha1.pdf

Cohen, R. J., Swerdlik, M. E., \& Sturman E. D. (2014). Testagem e avaliação psicológicas: Introdução a Testes e Medidas. (8a ed.). AMGH.

Dias, P. C., Henriques, P., Anjos, L. A. dos, \& Burlandy, L. (2017). Obesidade e políticas públicas: concepções e estratégias adotadas pelo governo brasileiro. Cadernos de Saúde Pública, 33(7). https://doi.org/10.1590/0102-311x00006016

Fereidouni, F., Atef-Vahid, M. K., Fathali Lavasani, F., Jamshidi Orak, R., Klonsky, E. D., \& Pazooki, A. (2014). Are Iranian obese women candidate for bariatric surgery different cognitively, emotionally and behaviorally from their normal weight counterparts? Eating and Weight Disorders - Studies on Anorexia, Bulimia and Obesity, 20(3), 397-403. https://doi.org/10.1007/s40519-014-0168-6

Fox, J. R. E., Msetfi, R. M., Johnson, R. S., \& Haigh, E. (2016). The Perception of Threat from Emotions in Predicting Binge Eating Behaviours in People Who Are Obese and Seeking Treatment for Their Weight. Clinical Psychology \& Psychotherapy, 23(5), 452-459. https://doi.org/10.1002/cpp.1972

Grothe, K. B., Mundi, M. S., Himes, S. M., Sarr, M. G., Clark, M. M., Geske, J. R., Kalsy, S. A., \& Frye, M. A. (2014). Bipolar disorder symptoms in patients seeking bariatric surgery. Obesity surgery, 24(11), 1909-1914. https://doi.org/10.1007/s11695-014-1262-6.

Hall, B. J., Hood, M. M., Nackers, L. M., Azarbad, L., Ivan, I., \& Corsica, J. (2013). Confirmatory factor analysis of the Beck Depression Inventory-II in bariatric surgery candidates. Psychological assessment, 25(1), 294-299. https://doi.org/10.1037/a0030305.

Hood, M. M., Grupski, A. E., Hall, B. J., Ivan, I., \& Corsica, J. (2013). Factor structure and predictive utility of the Binge Eating Scale in bariatric surgery candidates. Surgery for Obesity and Related Diseases, 9(6), 942-948. https://doi.org/10.1016/j.soard.2012.06.013

Iceta, S., Disse, E., Gouillat, C., Laville, M., Saoud, M., \& Robert, M. (2016). Personality but not Eating Behavior Is Different in Revisional Bariatric Surgery Candidates. Bariatric Surgical Practice and Patient Care, 11(4), 183-188. https://doi.org/10.1089/bari.2016.0028

Litwin, R., Goldbacher, E. M., Cardaciotto, L., \& Gambrel, L. E. (2016). Negative emotions and emotional eating: the mediating role of experiential avoidance. Eating and Weight Disorders - Studies on Anorexia, Bulimia and Obesity, 22(1), 97-104. https://doi.org/10.1007/s40519-016-0301-9

Mahony, D. (2010). Psychological Assessments of Bariatric Surgery Patients. Development, Reliability, and Exploratory Factor Analysis of the PsyBari. Obesity Surgery, 21(9), 1395-1406. https://doi.org/10.1007/s11695-010-0108-0

Marek, R. J., Tarescavage, A. M., Ben-Porath, Y. S., Ashton, K., Heinberg, L. J., \& Rish, J. M. (2017). Associations between psychological test results and failure to proceed with bariatric surgery. Surgery for Obesity and Related Diseases, 13(3), 507-513. https://doi.org/10.1016/j.soard.2016.09.007

Mendes, K. D. S., Silveira, R. C. de C. P., \& Galvão, C. M. (2008). Revisão integrativa: método de pesquisa para a incorporação de evidências na saúde e na enfermagem. Texto \& Contexto - Enfermagem, 17(4), 758-764. https://doi.org/10.1590/s0104-07072008000400018

Nicolau, I., Helena, F., Santo, E., Lube, C., \& Chibante, P. (2017). Tratamento multiprofissional para adultos obesos grau III Tratamiento multidisciplinario para los adultos obesos grado III Multidisciplinary treatment for third degree obese adults. Revista Cubana de Enfermería, 33(2), 386-403. http://scielo.sld.cu/pdf/enf/v33n2/enf18217.pdf

Oliveira, L. M. R., Vergara, C. M. A. C., Sampaio, H. A. de C., \& Vasconcelos Filho, J. E. de. (2018). Tecnologia mHealth na prevenção e no controle de obesidade na perspectiva do letramento em saúde: Lisa Obesidade. Saúde Em Debate, 42(118), 714-723. https://doi.org/10.1590/0103-1104201811814

Ortega, J., Fernandez-Canet, R., Alvarez-Valdeita, S., Cassinello, N., \& Baguena-Puigcerver, M. J. (2012). Predictors of psychological symptoms in morbidly obese patients after gastric bypass surgery. Surgery for obesity and related diseases: official journal of the American Society for Bariatric Surgery, 8(6), 770776. https://doi.org/10.1016/j.soard.2011.03.015.

Principais itens para relatar Revisões sistemáticas e Meta-análises: A recomendação PRISMA. (2015). Epidemiologia E Serviços de Saúde, 24(2), 335-342. https://doi.org/10.5123/s1679-49742015000200017

Pataky, Z., Carrard, I., Gay, V., Thomas, A., Carpentier, A., Bobbioni-Harsch, E., \& Golay, A. (2018). Effects of a Weight Loss Program on Metabolic Syndrome, Eating Disorders and Psychological Outcomes: Mediation by Endocannabinoids? Obesity facts, 11(2), 144-156. https://doi.org/10.1159/000487890.

Poubel. L. N., \& Rodrigues, P. (2018). Manual de inteligência psicológica para felicidade integral. Letras e Versos.

Ribeiro, G., Giapietro, H. B., Belarmino, L. B., \& Salgado-Junior, W. (2018). Depression, anxiety, and binge eating before and after bariatric surgery: problems that remain. Arquivos brasileiros de cirurgia digestiva: $A B C D=$ Brazilian archives of digestive surgery, 31(1), e1356. https://doi.org/10.1590/0102$672020180001 \mathrm{e} 1356$

Safer, D. L., Adler, S., \& Masson, P. C. (2020). Programa DBT para o comer emocional e compulsivo, Hofrefe.

Ganga, G. M. D., Soma, T. S., \& Hoh, G. D. (2012). Trabalho de conclusão de curso (TCC) em engenharia de produção. Atlas.

Schutt, P. E., Kung, S., Clark, M. M., Koball, A. M., \& Grothe, K. B. (2016). Comparing the Beck Depression Inventory-II (BDI-II) and Patient Health Questionnaire (PHQ-9) Depression Measures in an Outpatient Bariatric Clinic. Obesity surgery, 26(6), 1274-1278. https://doi.org/10.1007/s11695-015-1877-2.

McLean, R. C., Morrison, D. S., Shearer, R., Boyle, S., \& Logue, J. (2016). Attrition and weight loss outcomes for patients with complex obesity, anxiety and depression attending a weight management programme with targeted psychological treatment. Clinical obesity, 6(2), 133-142. https://doi.org/10.1111/cob.12136. 
Research, Society and Development, v. 11, n. 1, e21111125038, 2022

(CC BY 4.0) | ISSN 2525-3409 | DOI: http://dx.doi.org/10.33448/rsd-v11i1.25038

Siqueira A. C., \& Zanotti, S. V. (2017). Programa de Cirurgia Bariátrica e Reganho de Peso. Psicologia,Saúde e Doenças. Sociedade Portuguesa de Psicologia da Saúde - SPPS 18(1), 157-169. http://www.scielo.mec.pt/pdf/psd/v18n1/v18n1a14.pdf

World Health Organization. (2018). Obesity and overweight fact sheet. Home. Who.int. https://www.who.int 